\title{
The Value of Electron Microscopy in the Diagnosis of Chronic Renal Allograft Rejection
}

\author{
B. Ivanyi, M.D., E. Kemeny, M.D., E. Szederkenyi, M.D., F. Marofka, M.D., P. Szenohradszky, M.D. \\ Departments of Pathology (BI, EK) and Transplant Surgery (ES, MF, PS), University of Szeged, \\ Szeged, Hungary
}

The main causes of the late dysfunction of renal allografts are chronic rejection and chronic transplant nephropathy. Both are clinicopathologic entities, with a similar clinical presentation, but different histologic appearances. Chronic rejection is characterized by the presence of alloantigeninduced lesions (transplant arteriopathy and transplant glomerulopathy), and chronic transplant nephropathy by nonspecific sclerosing changes. The incidence of transplant arteriopathy and transplant glomerulopathy is relatively low. Electron microscopy (EM) may overcome the limitations in the histologic diagnosis of chronic rejection, because it verifies alloantigen-induced chronic microvasculopathy in the peritubular capillaries (transplant capillaropathy), and identifies transplant glomerulopathy more precisely than does light microscopy. To assess the value of EM in chronic rejection diagnosis, a retrospective search for transplant capillaropathy and transplant glomerulopathy was performed in a consecutive series of 91 biopsies performed $\geq 6$ months after implantation (median: 26 months, range 6-186) and the diagnoses were reclassified on the basis of the ultrastructural findings. The definitions used were: transplant capillaropathy: a peritubular capillary profile with seven or more circumferential basement membrane layers, or at least three profiles with five or six circumferential layers; ultrastructurally verified transplant glomerulopathy: thickening of the capillary wall in at least three loops in consequence of the widening of the subendothelial space by abnormal basement membrane material, and the formation of a new layer(s) of basal lamina; and chronic rejection: the presence of transplant capillaropathy and/or transplant glomerulopathy and/or transplant arteriopathy. Histologically, chronic transplant nephropathy,

Copyright (C) 2001 by The United States and Canadian Academy of Pathology, Inc.

VOL. 14, NO. 12, P. 1200, 2001 Printed in the U.S.A.

Date of acceptance: August 8, 2001.

Address correspondence to: Bela Ivanyi, Department of Pathology, University of Szeged, Kossuth L. sgt. 40, H-6724 Szeged, Hungary; e-mail: ivanyi@morpho.szote.u-szeged.hu; fax: 36-62-54-5868. chronic rejection, chronic cyclosporine nephrotoxicity, glomerulonephritis, acute rejection, "suspicious" for acute rejection, and "others" were diagnosed in $37 \%, 34 \%, 21 \%, 19 \%, 57 \%, 30 \%$, and $5 \%$ of the specimens, respectively. The results of EM increased the diagnosis of chronic rejection to $69 \%$ of the cases, and decreased chronic transplant nephropathy to $15 \%$. The individual incidence of transplant capillaropathy and transplant glomerulopathy was $79 \%$ and $57 \%$, respectively, and their cumulative incidence was $92 \%$. Five biopsies exhibited merely transplant arteriopathy. A late dysfunction typically had more than one cause; the most frequent combination was chronic rejection and acute rejection. In conclusion, the EM search for transplant capillaropathy and transplant glomerulopathy doubled the frequency of the diagnosis of chronic rejection. Currently, the evaluation of renal allograft biopsies from recipients with a late dysfunction relies on standard light microscopy. Because light microscopy per se proved to be insensitive in the diagnosis of chronic rejection, incorporation of EM into the evaluation of late dysfunction biopsies is strongly recommended.

KEY WORDS: Chronic allograft nephropathy, Electron microscopy, Diagnostic accuracy, Graft dysfunction, Peritubular capillary, Rejection, Transplant glomerulopathy.

Mod Pathol 2001;14(12):1200-1208

The long-term survival of renal allografts is influenced by alloantigen-dependent ("immunologic") factors and alloantigen-independent ("nephrondepleting") factors. The immunologic factors include the presence of panel-reactive antibodies, the quality of HLA match, the adequacy of immunosuppression, episodes of acute rejection, and drug compliance. Several factors of nephron depletion are known, such as advanced donor age, ischemiareperfusion injury, size mismatch, arterial hypertension, drug nephrotoxicity, lipid disorders, de novo or recurrent renal disease, hemodynamic overload with subsequent hyperfiltration injury to 
the glomeruli, etc. (1). The immunologic and nonimmunologic factors often act in parallel, and ultimately lead to a chronic graft dysfunction.

The analysis of biopsies from recipients with a chronic dysfunction (3 or more months after implantation) currently relies on standard light microscopy (2-5), and may reveal chronic rejection or chronic allograft nephropathy. Both are clinicopathologic entities, characterized clinically by a slow, insidious, progressive increase in the serum creatinine level, frequently in association with proteinuria and arterial hypertension. The diagnosis depends on whether lesions indicative of immunologic injury: transplant arteriopathy and/or transplant glomerulopathy are present in the biopsy specimen (chronic rejection) or not (chronic allograft nephropathy) (Table 1). In a recent review of 1500 allograft biopsies performed more than 6 months post-transplantation, 35\% of the cases were diagnosed as chronic allograft nephropathy, and $20 \%$ as chronic rejection (4). The histologic data suggest that chronic rejection is less frequent than chronic allograft nephropathy. However, the diagnosis of chronic rejection is often hampered because the pathologic features of rejection have an intrinsic focal nature (5), the fibrosing phase of transplant arteriopathy may be impossible to distinguish from intimal fibrosis induced by alloantigen-

TABLE 1. Definitions Used in the Present Study (Based on References 3, 4, 11, 17, and 18)

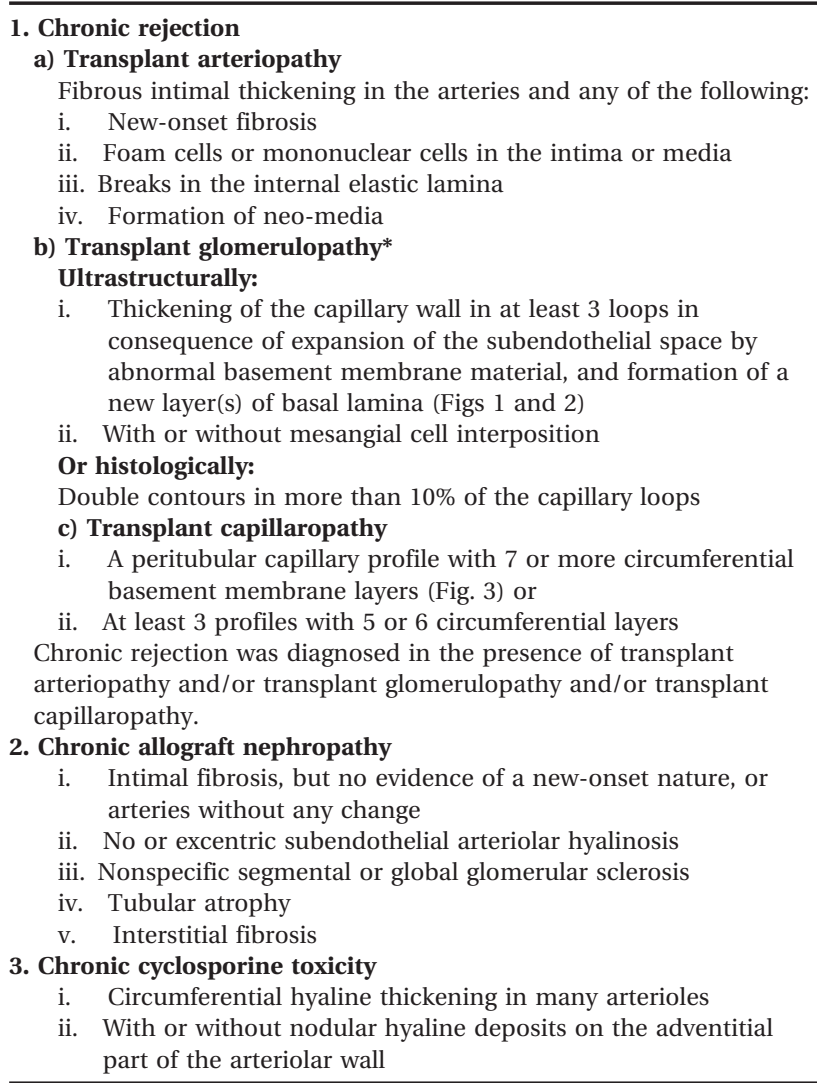

* Should be assessed in glomeruli not affected by segmental scarring or hyperfiltration. independent factors, and transplant glomerulopathy has a low incidence. The limitations in the histologic diagnosis of chronic rejection have resulted in unfavorable consequences. First, many pathologists indiscriminately use the terms "chronic rejection," "chronic transplant nephropathy," or "chronic transplant glomerulopathy" to label any sort of atrophy, hyalinosis, fibrosis or sclerosis in a kidney allograft biopsy, regardless of the etiology. Transplant clinicians are frustrated by the aimless and haphazard application of descriptive diagnostic categories that provide uncertainty rather than precision. Second, the lack of data on the true incidence of chronic rejection has generated a frustrating debate within the transplant community on the relative contributions of chronic rejection and nephron-depleting factors in long-term graft survival (6). Third, without a knowledge of the true incidence of chronic rejection, important therapeutic aspects of kidney transplantation, such as adequacy of immunosuppression, dose adjustment, a need to develop new immunosuppressive drugs, etc. cannot be analyzed properly.

The peritubular and glomerular capillary endothelial cells intensely express major histocompatibility complex class I and class II antigens, and are therefore targets of the rejection responses (7). Renal capillary pathology induced by rejection can better recognized ultrastructurally than by light microscopy. On electron microscopy (EM), the peritubular capillaries in grafts relating to a late dysfunction frequently exhibit circumferential multiplication and splitting of the basement membrane, and a nonfenestrated and thickened endothelial cell layer with a serrated contour along the interstitial aspect of the capillary profile (8-11). We recently validated these basement membrane alterations in the cortical labyrinth as an ultrastructural marker of chronic rejection in carefully selected series of chronic rejection and controls. Peritubular capillary profiles with several circumferential basement membrane layers occurred exclusively in chronic rejection (transplant capillaropathy; for a definition, see Table 1). We also experienced that EM of the glomeruli verified transplant glomerulopathy more precisely than did light microscopy (11). Pooled data indicate that acute rejection episodes or ongoing, subclinical rejection injuries to peritubular and glomerular capillary endothelial cells lead to the development of transplant capillaropathy and transplant glomerulopathy (11-16). The cumulative incidence of peritubular and glomerular capillary lesions was $85 \%$, suggesting that EM may be routinely used in the diagnosis of chronic rejection. The aim of the present study is to provide relevant data by comparing the diagnoses obtained by light microscopy per se, and by light microscopy supplemented with EM in a consecutive series of 91 allograft biopsies relating to a late dysfunction. By means of EM, transplant capillaropathy and transplant glomerulopathy were sys- 
tematically searched for retrospectively. To distinguish the ultrastructural features of transplant glomerulopathy from those induced by alloantigenindependent factors, a pilot study of the glomerular capillaries was carried out in native renal biopsies from patients with progressive kidney disease.

\section{MATERIALS AND METHODS}

In the Department of Pathology, University of Szeged, renal allograft biopsies from patients with a chronic allograft dysfunction are routinely investigated by the combined use of light microscopy (H\&E, periodic acid-Schiff, trichrome, methenamine silver and elastica) and immunofluorescence (IgG, IgA, IgM, C3, C1q and fibrinogen; since 1999 also with $\mathrm{C} 4 \mathrm{~d}$ ). Pieces of renal tissue are embedded for EM in each case. However, ultrastructural analysis is performed only if indicated as necessary by the clinical data and/or morphologic findings.

Between January 1, 1993 and June 1, 2000, 105 biopsies were performed 6 or more months posttransplantation. Ninety-one of these biopsies form the subject of the present study. The remainder were excluded because the resin-embedded tissue did not contain glomeruli, or the biopsies did not meet the criteria of specimen adequacy (10 or more glomeruli with at least two arteries). The donors and the recipients were all caucasian. All but one had received a cadaveric kidney. Eleven patients exhibited a poor initial graft function. They were treated with antilymphocyte drugs in the first 2 postoperative weeks. Basiliximab induction therapy was administered to four immunologically highrisk patients. Post-transplant immunosuppression was maintained with cyclosporine and prednisone in 47 recipients (including the basiliximab-treated recipients), with cyclosporine, prednisone and azathioprine in 18 recipients, and with cyclosporine, prednisone and mycophenolate mofetil in 16 recipients. The dose of cyclosporine was 3 to $5 \mathrm{mg} / \mathrm{kg} /$ day, adjusted individually according to the serum

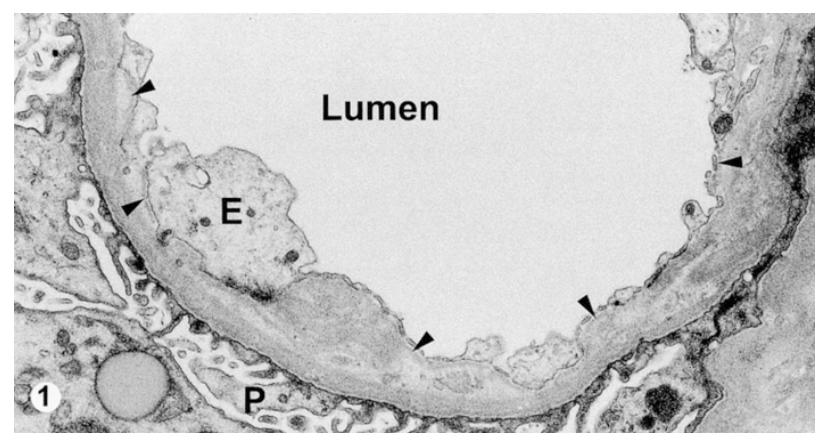

FIGURE 1. Transplant glomerulopathy. Accumulation of basement membrane material and presence of well-developed subendothelial basal lamina along the entire capillary circumference (arrowheads). E, endothelial cell; $P$, podocyte; Lumen, capillary lumen.

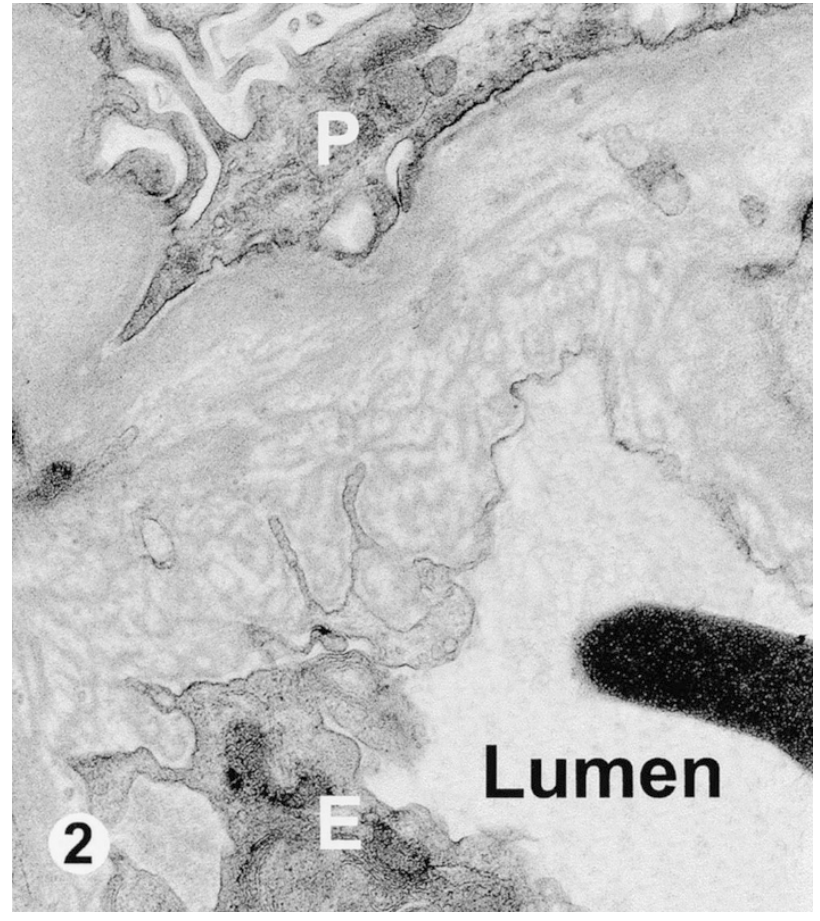

FIGURE 2. Transplant glomerulopathy. Multiplication and splitting of the subendothelial basal lamina. E, endothelial cell; $P$, podocyte; Lumen, capillary lumen.

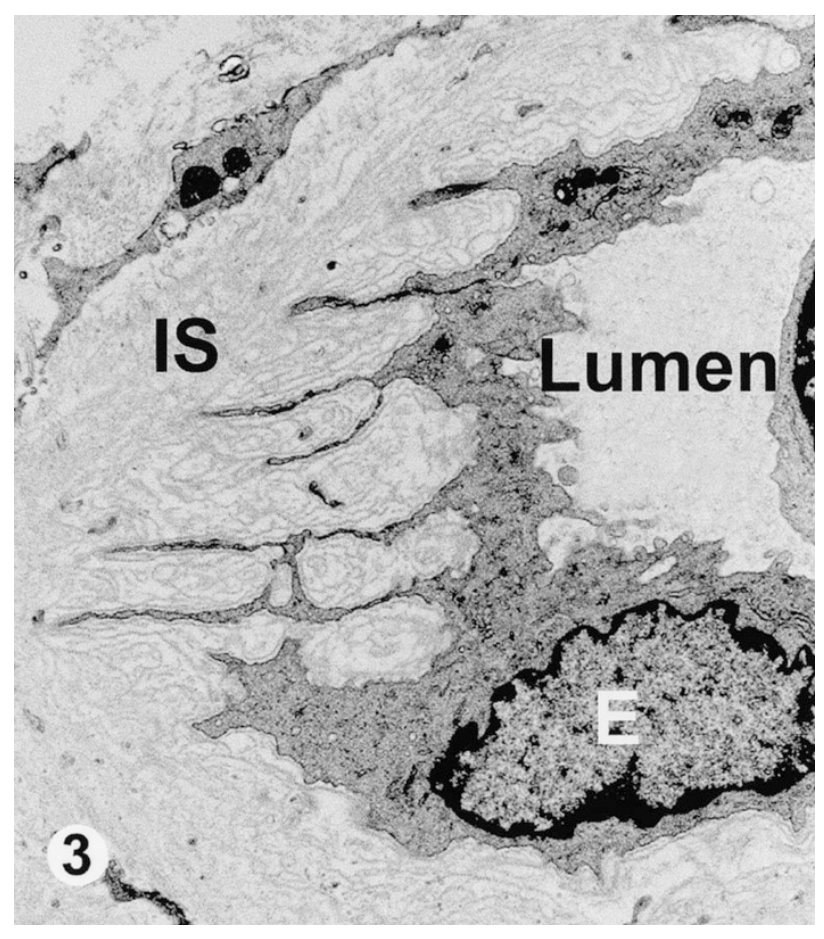

FIGURE 3. Transplant capillaropathy. A peritubular capillary profile with circumferential multiplication and splitting of the basement membrane. The number of layers is more than seven. The endothelium (E) is thickened and nonfenestrated and has a serrated contour. Lumen, capillary lumen; IS, peritubular interstitium.

cyclosporine level and the length of the posttransplantation period. The dose of mycophenolate mofetil was $2 \mathrm{~g} /$ day, but this was halved in five 


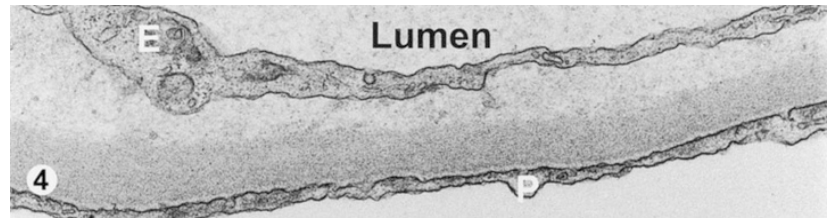

FIGURE 4. Thickened glomerular capillary from benign nephrosclerosis. Subendothelial expansion without newly formed subendothelial basal lamina.

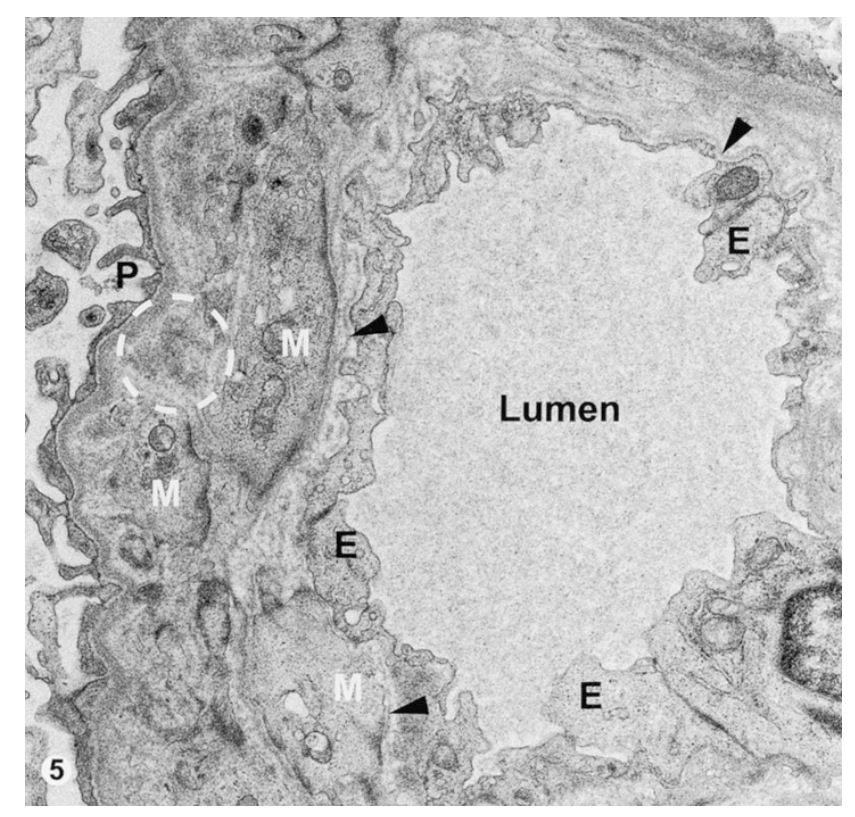

FIGURE 5. Thickened glomerular capillary from benign nephrosclerosis with hyperfiltration. Mesangial cell interposition (M), protein insudation (circle), and subendothelial basal lamina (arrowheads).

patients because of the side effects of the drug. Up to 1991, episodes of acute rejection were medicated with intravenous methylprednisolone for 3 to 5 days. Since 1991, steroid-resistant rejection has been treated with antilymphocyte drugs for 7 to 10 days. The grade of proteinuria at the time of the biopsy was tested with sulfosalicylic acid, and was evaluated as $0,1+, 2+, 3+$ and $4+$. Differences in grades between the various groups (see Results) were analyzed by the Wilcoxon signed rank test. Statistical significance was accepted at a probability level of $5 \%$. In our laboratory, a grading of $1+$ corresponded to proteinuria below $0.5 \mathrm{~g} / \mathrm{day}, 2+$ to
0.5-1 g/day, $3+$ to $1-2 \mathrm{~g} /$ day, and $4+$ to more than $2 \mathrm{~g} /$ day. Chronic obstructive uropathy was demonstrated by ultrasonography. The demographics, transplant-related variables and posttransplantation variables are shown in Table 2.

\section{Glomerular Capillary Ultrastructure in Native Kidney Biopsies}

The controls comprised 11 caucasian patients (seven men and four women; median age 45 years, range 29-68; median level of serum creatinine at biopsy: $1.68 \mathrm{mg} / \mathrm{dL}, 0.87-4.08)$. Seven patients suffered from hypertensive kidney disease, three patients from sclerosing IgA nephritis, and one patient from diabetic nodular glomerulosclerosis. On EM, glomeruli in seven specimens demonstrated signs of hyperfiltration, characterized by detachment of endothelial cells, "giant" subendothelial and/or intracapillary proteinaceous deposits, and densities in the glomerular basement membrane and paramesangium, with or without platelets and fibrin fibrils (19). The glomeruli in four specimens displayed segmental scarring with adhesion to the Bowman's capsule. The results of the pilot study are shown in Table 3.

\section{Study Design}

The biopsies were re-read and scored according to the Banff 1997 scheme (3). The lesions were categorized as "suspicious" for acute rejection, acute rejection, chronic rejection, chronic allograft nephropathy, chronic cyclosporine nephrotoxicity, glomerulonephritis, and "others." Acute rejection was subdivided into cell-mediated: tubulointerstitial rejection with or without intimal arteritis, and antibody-mediated: transmural arteritis with or without fibrinoid necrosis. The definitions of chronic rejection, chronic allograft nephropathy and chronic cyclosporine nephrotoxicity are given in Table 1. Two incidences of chronic rejection and chronic allograft nephropathy were calculated: one diagnosed by light microscopy per se, and one diagnosed by the combination of light microscopy and EM. Because it is not necessary to know the exact actual semiquantitative Banff scores to understand the results of the present article, the scores were simply categorized as no, mild, moder-

TABLE 2. Patient Characteristics

\begin{tabular}{lll}
\hline Males; females & $52 ; 29$ & $\geq 50: 14$ recipients \\
Donor age (years; median, range) & $41,8-60$ & $\geq 24: 14$ recipients \\
Cold ischemia (hours; median, range) & $21,10-29$ & $\geq 10: 7$ recipients \\
Incompatibility index for HLA ABDR (1-15; median, range)* & $7,1.5-14$ & $\geq 20 \%: 10$ recipients \\
Panel reactive antibody positivity & 19 recipients & \\
Recipient's age at biopsy (years; median, range) & $43,12-64$ & $26,6-186$ \\
Period between implantation and biopsy (months; median, range) & $4.17,1.62-12.4$ & \\
Serum creatinine at biopsy (mg/dL; median, range) & \\
\hline
\end{tabular}

*Score 1 indicates "full house" compatibility, and score 15 denotes no compatibility. 
TABLE 3. Thickening of the Glomerular Capillaries Related to Alloantigen-Independent Factors

1) Subendothelial expansion of capillary loops (Fig. 4)

i. Accumulation of abnormal basement membrane material in the lamina rara interna

ii. Bulging of endothelial cells (processes and/or nuclei) into the capillary wall

iii. No well-developed subendothelial basal lamina along the capillary circumference

2) Partial collapse of capillary loops

i. Detachment of foot processes

ii. Wrinkled lamina densa

iii. Occasional accumulation of abnormal basement membrane material in the lamina rara interna

iv. No well-developed subendothelial basal lamina along the capillary circumference

3) Capillary loops in the vicinity of hyperfiltration injury (Fig. 5)

i. Accumulation of subendothelial abnormal basement membrane material

ii. Duplicated or multiplicated subendothelial basal lamina

iii. Endothelial cells and/or mesangial cells in the capillary wall

iv. Electron-dense material in the capillary wall

ate, or severe lesions. The results of implantation biopsy were available in 28 recipients. For ultrastructural evaluation, the thin sections were placed on large slotted grids $(2 \times 1 \mathrm{~mm})$, and at least one glomerulus and all peritubular capillary profiles in the cortical labyrinth (i.e., capillaries around glomeruli) were sampled. The site of reading of the peritubular capillary basement membrane layers was the narrow peritubular interstitium. The multilayering of the basement membrane was regarded as circumferential if it was seen in at least three of the four portions of the capillary circumference (11). The median numbers of glomeruli and peritubular capillary profiles per examined biopsy were 1.8 (range: 1-4) and 15.5 (range: 6-32), respectively.

\section{RESULTS}

Table 4 shows the conditions after re-reading of the slides and consideration of the results of EM. As they are grouped, they do not represent the rank of impact with which they influenced the function of the graft. On EM, transplant capillaropathy and/or transplant glomerulopathy were observed in 20 cases of chronic allograft nephropathy, and in 12 cases of chronic cyclosporine toxicity, and these cases were reclassified as involving chronic rejection.

\section{Chronic Rejection}

Transplant capillaropathy was observed in 50 specimens, with the simultaneous presence of transplant glomerulopathy in 28 specimens. Transplant glomerulopathy was identified by EM in six specimens. EM confirmed histologically suspicious transplant glomerulopathy in 17 specimens. Eight biopsies displaying transplant glomerulopathy were negative for transplant capillaropathy. The in-
TABLE 4. Immunologic and Nonimmunologic Factors in Patients with a Late Graft Dysfunction

\begin{tabular}{lcc}
\hline Morphologic findings & LM & After EM \\
Chronic rejection & $31(34 \%)$ & $63(69 \%)$ \\
Chronic allograft nephropathy & $34(37 \%)$ & $14(15 \%)$ \\
Acute rejection & $52(57 \%)$ & \\
"Suspicious" for acute rejection & $27(30 \%)$ & \\
Chronic cyclosporine toxicity & $19(21 \%)$ & \\
Glomerulonephritis* & $17(19 \%)$ \\
Acute pyelonephritis & $2(2 \%)$ \\
Hypertension-related hyperplastic & $2(2 \%)$ \\
$\quad$ arteriolosclerosis & $1(1 \%)$ \\
Cholesterol microembolism & \\
Clinical factors of nephron depletion & $16(18 \%)$ \\
Hypertension ( $\geq 180 / 100$ mm Hg) & $8(9 \%)$ \\
Obstructive uropathy & $4(4 \%)$ \\
Diabetes & $1(1 \%)$ \\
Potential size mismatch & $1(1 \%)$ & \\
Nephrolithiasis &
\end{tabular}

*De novo 15: membranous 9, IgA 2, mesangial proliferative 2, focal sclerosis 1 , not otherwise specified 1; recurrent 2: focal sclerosis 1 , membranous nephropathy with anti-tubular basement membrane nephritis 1 . LM, light microscopy; EM, electron microscopy.

dividual frequency of transplant capillaropathy and transplant glomerulopathy was $79 \%$ and $57 \%$, respectively, whereas their cumulative incidence was $92 \%$. Five biopsies exhibited transplant arteriopathy and were negative for both transplant glomerulopathy and transplant capillaropathy.

\section{Coexisting Conditions}

\section{Acute Rejection}

The cell-mediated type was present in 50 specimens (tubulointerstitial: 27, arterial: 13), and the antibody-mediated type in two specimens.

\section{Nephron-Depleting Factors}

Cyclosporine toxicity coexisted on 12 occasions, glomerulonephritis on seven occasions and hyperplastic arteriolosclerosis associated with hypertension on two occasions. The group of glomerulonephritis comprised four cases of membranous nephropathy, two of mesangial proliferative glomerulonephritis, and one of immune complex glomerulonephritis, not otherwise specified. Two cases of membranous nephropathy also met the minimum ultrastructural criteria of transplant glomerulopathy.

Marked hypertension $(\geq 180 / 100 \mathrm{~mm} \mathrm{Hg}$ ) was recorded in nine patients, diabetes in three patients, the possibility of a size mismatch in one patient (donor: 8 years of age, recipient: 44 years of age), obstructive uropathy in three patients, and nephrolithiasis in one patient. In cases with obstructive uropathy, coexisting infected urine was verified in two patients. EM did not reveal diffuse glomerular basement membrane thickening as a sign of early diabetic nephropathy in any of the diabetics. In the case involving a potential size mismatch, diffuse lamellation of the glomerular base- 
ment membrane, reported in renal allografts from some pediatric donors to adult recipients (20), was not observed on EM. Three or more peritubular capillary profiles with five or six circumferential layers were observed in two patients with obstructive uropathy and in the nephrolithiatic patient. Besides transplant capillaropathy, these biopsies also revealed other morphologic features of chronic rejection. The biopsy specimen of the third patient with obstructive uropathy displayed transplant glomerulopathy, and one peritubular capillary exhibited five or six circumferential layers.

\section{Chronic Rejection and Newer Generations of Immunosuppressive Drugs}

Biopsies from three patients treated with basiliximab, and 13 patients treated with mycophenolate mofetil showed features of chronic rejection.

\section{Proteinuria}

The grade of proteinuria was analyzed after exclusion of the glomerulonephritic cases and cases with urinary tract infection. Three groups were formed on the basis of the presence and the severity of transplant glomerulopathy: no glomerulopathy (median 2, range $0-3$ ), ultrastructurally verified or confirmed glomerulopathy (median 2, range $0-3$ ), and histologically moderate or severe glomerulopathy (median 3, range $0-4$ ). The differences were not significant, though the difference in the grade of proteinuria between the group without glomerulopathy and the group with histologically moderate or severe glomerulopathy was close to the level of significance $(P=.07)$.

\section{Follow-Up}

Two patients with a good renal function died within 12 months after biopsy. Thirty-four grafts terminated in chronic renal failure necessitating hemodialysis after a median postbiopsy period of 4.5 months. Twenty-four grafts are still functioning (median postbiopsy period: 12 months), with a serum creatinine level below $3.95 \mathrm{mg} / \mathrm{dL}$ in 20 patients. Follow-up data were not available on three patients.

\section{Chronic Allograft Nephropathy}

There was no intimal fibrosis in five specimens. Mild or moderate intimal fibrosis was recorded in nine specimens. Twelve biopsies had at least moderate tubular atrophy and interstitial fibrosis.

Acute rejection coexisted in all biopsies ("suspicious" for acute rejection: 2). Three specimens showed membranous nephropathy 3 , and one focal sclerosis.
Clinically, four patients were markedly hypertensive ( $\geq 180 / 100 \mathrm{~mm} \mathrm{Hg}$ ), and two patients had obstructive uropathy. One patient with obstructive uropathy also had an active urinary tract infection. On EM, cases with obstructive uropathy displayed a peritubular capillary profile with five or six circumferential basement membrane layers. After exclusion of the glomerulonephritic cases and obstructive uropathy with coexisting infected urine, the median grade of proteinuria was 2 (range $0-4$ ).

Three patients treated with mycophenolate mofetil developed chronic allograft nephropathy, as did one patient treated with basiliximab.

Eight patients returned to hemodialysis (median postbiopsy period: 8.5 months). Six grafts are functioning with acceptable parameters in three patients (follow-up: within 8 months).

\section{Chronic Cyclosporine Toxicity}

This was diagnosed in 19 biopsy specimens. Seven cases lacked coexisting lesions of chronic rejection. These were associated with "suspicious" for acute rejection in four specimens, IgA glomerulonephritis in two specimens, and cholesterol microembolism in one specimen.

The clinical "load" factors in the seven cases included blood pressure levels of 200/100 mm Hg in two patients, and diabetes in one patient. EM did not reveal early diabetic glomerulopathy.

One of the IgA nephritic patients died with a functioning graft 15 months after biopsy; the other returned to hemodialysis 32 months after biopsy. The remaining four patients have an acceptable renal function (follow-up: within 9 months).

\section{Acute Rejection}

Two cases manifested late acute rejection. "Suspicious" for acute rejection developed in one patient, and acute tubulointerstitial rejection and membranous nephropathy in the other patient. Both patients responded to anti-rejection therapy. The patient with borderline rejection has a good graft function 10 months after biopsy. Graft loss occurred in the glomerulonephritic patient 1 year after the biopsy.

\section{Others}

Six cases belonged in this group. One case exhibited no abnormality (serum creatinine $1.62 \mathrm{mg} / \mathrm{dL}$, seropositive for hepatitis $C$ virus), two cases acute pyelonephritis, and three cases glomerulonephritis. The glomerulonephritic cases included membranous nephropathy, recurrent focal segmental glomerulosclerosis and recurrent anti-tubular basement membrane nephritis associated with immune complex glomerulonephritis. The latter case did not 
recur for 2 years after transplantation (21). Coexisting obstructive uropathy was observed in the cases with pyelonephritis, and in the case of membranous nephropathy. None of them displayed a peritubular capillary profile with five or six circumferential basement membrane layers. All grafts are functioning, with acceptable parameters in four patients (follow-up: up to 12 months).

\section{DISCUSSION}

\section{Ultrastructural Markers of Chronic Rejection}

\section{Transplant Capillaropathy}

This was frequently observed $(79 \%)$ in the present series involving chronic rejection. To date, 227 cases of native kidney disease have been analyzed in the literature for the presence of circumferential basement membrane layers in cortical peritubular capillaries, including hypertension, diabetes, chronic cyclosporine toxicity, sclerosing glomerulonephritis, chronic tubulointerstitial nephritis, thrombotic microangiopathy, cryoglobulinemia, primary glomerulonephritides, lupus nephritis, and kidney diseases in elderly people (911). A peritubular capillary profile with seven or more circumferential layers or at least three profiles with five or six circumferential layers was not observed in these disorders, but only in chronic rejection. Verification of transplant capillaropathy is of great value, because it permits the diagnosis of chronic rejection even in the presence of various nephron-depleting factors. A few conditions, however, should be borne in mind during the diagnosis of transplant capillaropathy. Two capillary profiles with five or six layers have been very occasionally observed in chronic tubulointerstitial nephritis and hemolytic-uremic syndrome, and marked basement membrane multiplication has been noted in a biopsy specimen of native end-stage kidney from an adult with reflux nephropathy (11). Because cases with reflux nephropathy are seldom biopsied, data on peritubular capillary changes in this condition are lacking. The situation is similar in chronic obstructive nephropathy. In the present study, cortical peritubular capillaries were examined in nine biopsies from patients with chronic obstructive uropathy, including the patient with a renal stone. Transplant capillaropathy was present only in those three specimens that also exhibited other morphologic features of chronic rejection. We conclude, therefore, that obstructive uropathy before the endstage kidney phase does not appear to be associated with the development of a transplant capillaropathy-like lesion. In the event of a history of obstructive uropathy or thrombotic microangiopathy, however, the presence of transplant capillaropathy must be interpreted with caution.
Peritubular capillary endothelial cells are targets of rejection responses. Acute humoral rejection is characterized by the linear immunofluorescence of complement $4 \mathrm{~d}$ along the capillary walls (16), and the necrosis of endothelial cells and denudation of the basement membrane observed on EM (22). The ultrastructural features of acute cellular rejection include hypertrophied endothelial cells, increased adherence and passage of lymphocytes, lymphocytes in the capillary wall, separation of endothelial cells from the basement membrane, defects in the endothelial lining and balloon-like fragmentation or apoptosis of endothelial cells in the vicinity of lymphocytes $(12,23,24)$. In chronic rejection, the wall of the peritubular capillaries frequently contained lymphocytes, separating the endothelial cells from the laminated basement membrane (11). The observations suggest that transplant capillaropathy is the final result of ongoing or repeated episodes of endothelial cell damage or death and subsequent regeneration. The process is associated with a loss of fenestration, a serrated shape, and splitting and multilayering of the basement membrane. In semithin sections, the capillaropathy appears as a serrated profile with a thick, ribbon-like basement membrane layer (11), enhancing the sampling of the lesion. Transplant capillaropathy may be encountered sooner than 6 months after transplantation. We have seen two cases, not included in the present study, which exhibited the lesion 3.5 months and 4 months postimplantation, respectively. One of the recipients received basiliximab, and the other was treated with mycophenolate mofetil.

\section{Transplant Glomerulopathy}

This is a traditional histologic marker of chronic rejection (3), but its incidence is low, ranging between $15 \%$ and $30 \%(5,25)$. The morphology of glomerulopathy resembles that seen in renal thrombotic microangiopathy or antiphospholipid syndrome $(26,27)$, suggesting that humorally mediated endothelial damage may play a role in the evolution of the lesion. The light microscopic distinction between the presence or absence of transplant glomerulopathy may occasionally be difficult. We (11) and others $(9,28-30)$ have experienced that EM is a reliable tool with which to verify histologically suspicious transplant glomerulopathy. However, there are no agreed ultrastructural criteria of transplant glomerulopathy, and the investigators did not define precisely what they regarded as the minimum lesion of transplant glomerulopathy. Glomerular basement membrane changes induced by alloantigen-independent factors should be excluded during the ultrastructural verification of transplant glomerulopathy. Our pilot study revealed that hyperfiltration injury-induced glomeru- 
lar basement membrane alterations or the collapse of capillary loops can easily be distinguished from transplant glomerulopathy. In contrast with a previous report (29), we do not regard subendothelial expansion without well-developed new subendothelial basal lamina involving the entire capillary circumference as a feature of transplant glomerulopathy, because subendothelial expansion alone was present in hemodynamically maladapted glomeruli in the present study, and in acute rejection in a previous communication (23). This experience led us to utilize the duplication of the glomerular basement membrane and cutoffs for the minimum level of affection of the capillary loops in the ultrastructural definition of transplant glomerulopathy. We were interested in learning whether cases with no glomerulopathy and ultrastructurally verified or confirmed glomerulopathy exhibit different proteinuria levels. No differences were observed, and the testing of different cutoffs (five affected capillaries, for instance, as opposed to the three used here) in association with the proteinuria level would therefore not be conclusive. Testing of mean values of 24-hour protein excretion with different cutoffs might well have led to some other conclusion about the optimal cutoff for the ultrastructural criterion of transplant glomerulopathy, but exact determination of the proteinuria level was not included in the protocol of patient evaluation at the time of the study period. In summary, when read according to rigorous criteria, ultrastructurally verified transplant glomerulopathy can be used as an indicator of chronic rejection, unless there is a history of thrombotic microangiopathy after transplantation.

\section{EM in the Evaluation of Late Dysfunction Biopsies}

In our series, the correct interpretation of glomerular lesions necessitated the examination of allograft biopsies by EM in $50 \%$ of the cases. The cases reclassified as having chronic rejection comprised 17 specimens with histologically suspicious transplant glomerulopathy. To categorize these cases properly, we relied on the study of the glomeruli by EM to be conclusive. The precise classification of cases with glomerulonephritis, the exclusion of early diabetic glomerulopathy and the study of the glomerular basement membrane in the pediatric donor kidney transplanted into an adult recipient needed the ultrastructural analysis of the glomeruli in $24 \%$ of the specimens. Our favorable experience with the diagnostic aid of EM is similar to that in a previous article, in which EM was estimated to be a relevant diagnostic tool in about half of the renal allograft biopsies (29). In the present study, a late dysfunction typically had more than one cause: the most frequent combination was chronic rejection and acute rejection, but we also observed chronic rejection, acute rejection and cyclosporine toxicity, or chronic rejection, acute rejection and glomerulonephritis, with or without clinically existing nephron-depleting factors. Similar experience has been reported by others $(4,25)$. It is our opinion that combinations of various forms of rejection and nephron-depleting factors can be interpreted best if light microscopy, immunofluorescence and EM are routinely applied. The verification of ultrastructural lesions of chronic rejection in the present study doubled the incidence of chronic rejection, and eliminated the excess diagnosis of chronic allograft nephropathy. Extrapolation of our data indicates that chronic rejection is markedly underdiagnosed by standard light microscopy per se. The ultrastructural search for transplant capillaropathy and glomerulopathy in late dysfunction biopsies with a light microscopic diagnosis of chronic allograft nephropathy is therefore recommended. There are arguments, however, which do not favor the incorporation of EM among the diagnostic modalities during the evaluation of late dysfunction biopsies: the ultrastructural verification of chronic rejection is time-consuming, the costs of EM are not negligible, and there is no specific therapy for chronic allograft nephropathy or chronic rejection. Because these objections are valid, nephropathology units should decide individually how to evaluate graft specimens relating to a late dysfunction.

\section{Chronic Rejection and Newer Immunosuppressive Drugs}

The findings presented here are in part based on a regimen of immunosuppressive therapy no longer widely used. Mycophenolate mofetil, as elsewhere, has replaced azathioprine in the protocol at our center, and a small number of immunologically high-risk patients received anti-CD25 antibody prophylaxis. The more recent generations of drugs, such as tacrolimus, sirolimus, mycophenolate mofetil and anti-CD25 antibodies, significantly reduce the likelihood of clinical acute rejection. It is of importance to document, therefore, what happens to these recipients years after implantation. Do these drugs eliminate chronic rejection? In a majority of our limited number of patients treated with such newer drugs, the answer was no. Because no method other than EM exists at the moment with which to verify chronic rejection precisely, its use is indispensable for those who wish to examine the influence of new immunosuppressive drugs on the prevention of chronic rejection. 


\section{CONCLUDING REMARKS}

If light microscopy alone is used to explore the cause of a late dysfunction, chronic rejection is underdiagnosed and chronic allograft nephropathy is overdiagnosed. Internationally accepted classifications on renal allograft pathology are encouraged to include EM in the routine evaluation of allograft biopsies to achieve better recognition of capillary lesions of chronic rejection.

Acknowledgments: Supported by grants to B. Ivanyi (OTKA T-025590, and ETT-588/1996-06, Budapest, Hungary). The comments of the reviewers are greatly appreciated.

\section{REFERENCES}

1. Halloran PF, Melk A, Barth C. Rethinking chronic allograft nephropathy: the concept of accelerated senescence. J Am Soc Nephrol 1999;10:167-81.

2. Colvin RB, Cohen A, Siaonz C, Bonsib S, Buick M, Burke B, et al. Evaluation of the pathologic criteria for acute renal allograft rejection: reproducibility, sensitivity and clinical correlation. J Am Soc Nephrol 1997;8:1930-41.

3. Racusen LC, Solez K, Colvin RB, Bonsib SM, Castro M, Cavallo $\mathrm{T}$, et al. The Banff 97 working classification of renal allograft pathology. Kidney Int 1999;55:713-23.

4. Mihatsch MJ, Nickeleit V, Gudat F. Morphologic criteria of chronic renal allograft rejection. Transplant Proc 1999;31: 1295-7.

5. Monaco AP, Burke JF, Ferguson RM, Halloran PF, Kahan BD, Light JA, et al. Current thinking on chronic renal allograft rejection: issues, concerns and recommendations from a 1997 roundtable discussion. Am J Kidney Dis 1999;33:150-60.

6. Matas AJ, Gillingham KJ, Humar A, Dunn DL, Sutherland DER, Najarian JS. Immunologic and nonimmunologic factors: different risks for cadaver and living donor transplantation. Transplantation 2000;69:54-8.

7. Fuggle SV, Errasti P, Daar AS, Fabre JW, Ting A, Morris PJ. Localization of major histocompatibility complex (HLA-ABC and DR) antigens in 46 kidneys. Transplantation 1983;35: 385-90.

8. Monga G, Mazzucco G, Novara R, Reale L. Intertubular capillary changes in kidney allografts: an ultrastructural study in patients with transplant glomerulopathy. Ultrastruct Pathol 1990;14:201-9.

9. Drachenberg CB, Steinberger E, Hoehn-Saric E, Heffes A, Klassen DK, Bartlett ST, et al. Specificity of intertubular capillary changes: comparative ultrastructural studies in renal allografts and native kidneys. Ultrastruct Pathol 1997;21:227-33.

10. Oikawa T, Morozumi K, Koyama K, Takeuchi O, Takeda A, Uchida K, et al. Electron microscopic peritubular capillary lesions: a new criterion for chronic rejection. Clin Transplantation 1999;13(Suppl. 1):24-32.

11. Ivanyi B, Fahmy H, Brown H, Szenohradszky P, Halloran PF, Solez K. Peritubular capillaries in chronic renal allograft rejection: a quantitative ultrastructural study. Hum Pathol 2000;31:1129-38.

12. Shimizu A, Colvin RB, Yamanaka N. Rejection of peritubular capillaries in renal allo- and xeno-graft. Clin Transplantation 2000;14(Suppl. 3):6-14.

13. Shirwan H. Chronic allograft rejection. Transplantation 1999;68:715-26.

14. Rush DN, Karpinski ME, Nickerson P, Dancea S, Birk P, Jeffery JR. Does subclinical rejection contribute to chronic rejection in renal transplant patients? Clin Transplantation 1999;13:441-6.

15. McKenna RM, Takemoto SK, Terasaki PI. Anti-HLA antibodies after solid organ transplantation. Transplantation 2000; 69:319-26.

16. Lederer SR, Kluth-Pepper B, Schneeberger H, Albert E, Land W, Feucht HE. Impact of humoral alloreactivity early after transplantation on the long-term survival of renal allografts. Kidney Int 2001;59:334-41.

17. Lipkowitz GS, Madden RL, Mulhern J, Braden G, O’Shea M, O'Shaughnessy J, et al. Long-term maintenance of therapeutic cyclosporine levels leads to optimal graft survival without evidence of chronic nephrotoxicity. Transpl Int 1999;12:202-7.

18. Mihatsch MJ, Antonovych T, Bohman S-O, Habib R, Helmchen U, Noel LH, et al. Cyclosporin. A nephropathy: standardization of the evaluation of kidney biopsies. Clin Nephrol 1994;41:23-32.

19. Rennke HG, Anderson S, Brenner BM. Structural and functional correlations in the progression of kidney disease. In: Tisher CC, Brenner BM, editors. Renal pathology with clinical and functional correlations. Philadelphia: Lippincott; 1989. pp. 43-66.

20. Nadasdy T, Abdi R, Pitha J, Slakey D, Racusen L. Diffuse glomerular basement membrane lamellation in renal allografts from pediatric donors to adult recipients. Am J Surg Path 1999;23:437-42.

21. Ivanyi B, Haszon I, Endreffy E, Szenohradszky P, Petri IB, Kalmar T, et al. Childhood membranous nephropathy, circulating antibodies to the $58-\mathrm{kD}$ TIN antigen and antitubular basement membrane nephritis: a follow-up during 11 years. Am J Kidney Dis 1998;32:1068-74.

22. Lajoie G. Antibody-mediated rejection of human renal allografts: an electron microscopic study of peritubular capillaries. Ultrastruct Pathol 1997;21:235-42.

23. Olsen ST. Pathology of allograft rejection. In: Burdick JF, Racusen LC, Solez K, Williams GM, editors. Kidney transplant rejection, 2nd ed. New York: Marcel Dekker; 1992. pp. 333-57.

24. Ivanyi B, Hansen HE, Olsen ST. Postcapillary venule-like formation of peritubular capillaries in acute renal allograft rejection. Arch Pathol Lab Med 1992;116:1062-7.

25. Morozumi K, Oikawa T, Fukuda M, Sugito K, Takeuchi O, Usami T, et al. Electron-microscopic peritubular capillary lesion is a specific and useful diagnostic indicator for chronic rejection of renal allografts showing less specific morphologic lesions in the cyclosporine era. Transplant Proc 1997;29:89-92.

26. Nochy D, Daugas E, Droz D, Beaufils H, Grünfeld J-P, Piette $\mathrm{J}-\mathrm{C}, \mathrm{B}$ et al. The inrarenal vascular lesions associated with primary antiphospholipoid syndrome. J Am Soc Nephrol 1999;10:507-18.

27. Baid S, Pascual M, Williams WW, Tolkoff-Rubin N, Johnson $\mathrm{SM}$, Collins B, et al. Renal thrombotic microangiopathy associated with anticardiolipin antibodies in hepatitis C-positive renal allograft recipients. J Am Soc Nephrol 1999; 10:146-53.

28. Monga G, Mazucco G, Messina M, Motta M, Quaranta S, Novara R. Intertubular capillary changes in kidney allografts: a morphologic investigation on 61 renal specimens. Mod Pathol 1992;5:125-30.

29. Herrera GA, Isaac J, Turbat-Herrera EA. Role of electron microscopy in transplant renal pathology. Ultrastruct Pathol 1997;21:481-98.

30. Morozumi K, Oikawa T, Fukuda M, Sugito K, Takeuchi O, Oda A, et al. Diagnosis of chronic rejection using peritubular and glomerular capillary lesions. Transplant Proc 1996;28: 508-11. 